\title{
Désir et affects incarnés auprès de migrants
} homosexuels mexicains

Le cas du groupe «Arc-en-ciel à Paris » à la Cité Internationale Universitaire de Paris

\section{Gerardo Perfors-Barradas}

\section{(2) OpenEdition}

Journals

Édition électronique

URL : http://journals.openedition.org/agedor/4662

DOI : 10.4000/agedor.4662

ISSN : 2104-3353

Éditeur

Laboratoire LISAA

Référence électronique

Gerardo Perfors-Barradas, "Désir et affects incarnés auprès de migrants homosexuels mexicains 》, L'Âge d'or [En ligne], 12 | 2019, mis en ligne le 01 octobre 2020, consulté le 14 octobre 2020. URL: http://journals.openedition.org/agedor/4662 ; DOI : https://doi.org/10.4000/agedor.4662

Ce document a été généré automatiquement le 14 octobre 2020

L'Âge d'or. Images dans le monde ibérique et ibéricoaméricain 


\section{Désir et affects incarnés auprès de migrants homosexuels mexicains}

Le cas du groupe « Arc-en-ciel à Paris » à la Cité Internationale

Universitaire de Paris

Gerardo Perfors-Barradas

\section{Introduction}

1 L'objet des sciences sociales, selon Maurice Godelier dans Au fondement des sociétés humaines, est « d'analyser et comprendre les conditions d'apparition et de disparition des différentes façons d'organiser la vie en société, des diverses manières de penser et d'agir, qui sont les racines de la diversité des formes connues d'identités individuelles et collectives, celles qui coexistent aujourd'hui et celles qui ont disparu $»^{1}$.

2 Prenant cela en compte, on peut rattacher à cette définition l'idée de l'homosexualité actuellement dominante dans le monde occidental contemporain, qui la voit comme une orientation sexuelle, souvent assimilée à une identité plutôt fixe, et en opposition à l'hétérosexualité. Cette notion et le terme même d'homosexualité sont apparus dans un contexte historique précis de changement du contrôle social sur la vie sexuelle. L'anthropologue Richard Parker', s'appuyant sur le travail capital de l'historien John D'Emilio ${ }^{3}$ cite l'urbanisation et l'industrialisation comme des facteurs clé, car l'une offre un cadre d'anonymat et l'autre réduit l'importance de la division sexuelle du travail. La possibilité de migrer, elle aussi, fait partie des conditions d'apparition dudit mode de vie. La transmission d'images par les médias de masse et les réseaux sociaux, l'augmentation des vols et la réduction de leur prix ${ }^{4}$, et les différences en termes de salaires et droits sociaux, font partie des conditions qui permettent la migration internationale.

Dans le cas des Mexicains, celle-ci a été historiquement dirigée vers les États-Unis, mais depuis le tournant du siècle de plus en plus vers l'Europe et la France aussi. Pour les personnes identifiées comme homosexuelles, les premiers exemples de ce mode de vie, distinct de celui des "vestidas", venaient de l'Europe et des États-Unis, où des activistes 
se sont par la suite éloignés du terme "homosexuel », considéré comme clinique, en faveur de "gay" et "queer", qui étaient utilisés par les gens en question". La séparation avec les réseaux de soutien familial pousse vers des «familles choisies» ou ce que l'on appelle la "queer kinship", différente de la norme hétéronucléaire ${ }^{7}$.

4 Cet article propose d'étudier le rôle des affects et du désir dans le phénomène de mobilité des hommes gay mexicains entre le Mexique et Paris, avec une approche qualitative, réflexive, (auto)ethnographique. Une partie importante des enquêtés, et celle qui sera traitée dans cet article, habite ou a habité plus précisément dans la Cité Internationale Universitaire de Paris (CIUP). Cet endroit conçu après la première guerre mondiale par le gouvernement français en collaboration avec des magnats industriels réunit plus de 40 maisons qui hébergent 6000 étudiants, chercheurs, artistes et sportifs de France et du monde dans un parc au sud de Paris.

Au sein de la Maison du Mexique de la cité universitaire, un groupe d'hommes gay s'est constitué informellement, avec le nom "Arcoíris en París" (arc-en-ciel à Paris). Par le biais de l'observation participante, des entretiens, et une méthodologie située féministe ${ }^{8}$, qui implique une prise de conscience des inégalités de genre et de leur impact, non seulement dans la société en général mais aussi dans les processus de production scientifique, j'ai pu observer la façon dont ces hommes incarnent leur parcours migratoire et jusqu'à quel point on peut parler d'une resignification des pratiques et de représentations de la sexualité dans le cadre spécifique de la Maison du Mexique et de la Cité Universitaire. Tout d'abord, on expliquera la méthodologie ; puis, on montrera des exemples de la resignification des notions de race, culture, et sexe parmi mes enquêtés; on évoquera certaines pratiques observées, comme le changement de comportements sexuels et du désir, la solidarité, mais aussi la reproduction de comportements discriminatoires, ainsi que les réactions face à l'éloignement de la famille dans le contexte d'une homophobie et une xénophobie persistantes. Dans cette analyse, on tiendra compte des différences d'origine sociale, ethnique et géographique des enquêtés, ainsi que du rôle du capital symbolique dans leurs trajectoires de mobilité.

\section{Une approche réaliste-critique pour une ethnographie réflexive}

6 La perspective qualitative, réflexive, (auto)ethnographique requiert une emphase sur le côté significatif de l'expérience des groupes humains étudiés, ainsi qu'une réflexion sur la position du chercheur en tant que sujet dans la constitution des données scientifiques issues d'une interprétation de la réalité empirique ${ }^{9}$. Elle s'inscrit dans le courant théorique du réalisme critique, qui prétend surmonter les limitations des approches positiviste et "interprétativiste», en proposant des recherches ethnographiques réflexives mais réalistes, qui produisent des explications ouvertes au débat et à la critique et qui offrent une meilleure compréhension qualitative des sociétés et des cultures humaines ${ }^{10}$. Cette approche implique une réflexion sur l'influence inévitable du corps et l'histoire personnelle du chercheur dans le travail de recherche. Pour cette recherche, notre parcours personnel, qui comprend une migration vers l'Europe depuis le Mexique datant du début du siècle nous a permis l'identification des phénomènes sociaux et historiques évoqués dans cet article, et qui n'ont pas encore été pleinement travaillés scientifiquement. Par ailleurs, les 
connaissances sociales, linguistiques et scientifiques acquises lors de ce parcours migratoire, culturel et universitaire ont été indispensables pour aborder et obtenir la confiance des enquêtés, malgré le fait que cette confiance ne soit pas absolue ni permanente, comme elle ne l'est jamais dans aucune relation. Une entrave occasionnelle dans ces relations établies, au-delà de celles inhérentes à l'étude d'un sujet parfois intime et tabou comme celui qui nous occupe, fut celle de la mise en question de notre pleine appartenance au groupe étudié, du fait de notre implantation en Europe avec droit de séjour permanent, ainsi que de notre apparence physique qui est moins susceptible d'être racialisée que celle de la plupart des enquêtés. Les techniques adoptées pour répondre à ces entraves furent celles de garantir pleinement l'anonymat des informations partagées, ainsi qu'un positionnement normatif explicite contre les inégalités structurelles de tout type.

\section{Terrain : critères de délimitation}

7 L'objectif des fondateurs de la Cité Internationale Universitaire de Paris, parmi lesquels se trouvaient des grands hommes d'affaires du début $d u x^{e}$ siècle et certains gouvernants européens et américains de l'entre-deux-guerres, était de créer une élite internationale pour la paix mondiale. Par conséquent, la plupart des maisons qui s'y trouvent sont associées à un pays. Parmi les pays latino-américains, le Mexique, le Brésil et l'Argentine ont leurs propres maisons, financées par leurs États respectifs. La famille Abreu de Grancher a financé une maison pour les étudiants cubains mais le gouvernement cubain a été écarté de la gestion de la maison par la voie légale suite au changement de régime en 1959.

8 Les revenus pétroliers ont eu un rôle important dans le développement de cette Cité. En effet, deux des mécènes les plus impliqués dans ce projet, Émile Deutsch de la Meurthe et John D. Rockefeller doivent leurs fortunes au pétrole. En ce qui concerne la maison du Mexique, financée par l'État mexicain, il est important de rappeler l'importante contribution de Petróleos Mexicanos au budget de l'État à partir de sa nationalisation en $1938^{11}$.

9 La maison du Mexique a été inaugurée en 1953. Comme indiqué dans l'inscription de la façade principale : “TESTIMONIO PERDURABLE DE LA AMISTAD ENTRE MÉXICO Y FRANCIA, ESTE EDIFICIO CONSTRUIDO PARA LA JUVENTUD ESTUDIOSA"12. Il faut dire que, dans sa conception, cette Cité était conçue principalement pour des étudiants hommes, et les femmes en étaient largement absentes. Aujourd'hui, les femmes sont beaucoup plus présentes sur le campus, sans qu'on puisse pour autant dire que l'espace a été libéré des conditions patriarcales dans lesquelles il a été conçu. Le bâtiment comporte une aile principale de 5 étages, et une annexe plus petite de 3 étages, cachée derrière la maison. Cette annexe était la partie destinée aux femmes, et cette ségrégation persiste jusqu'aux évènements de mai 1968, quand les étudiants ont réussi à y mettre fin ${ }^{13}$. Dans le hall, entre la bibliothèque et les bureaux administratifs, il y a un mur avec les portraits de résidents dits " célèbres ", quasi-exclusivement des hommes. Aujourd'hui, cependant, environ la moitié de la petite centaine de résidents est constituée de femmes et, parmi les hommes, il y a une grande proportion de personnes qui s'assument comme homosexuels, gays, ou bisexuels, entre 10 et $20 \%$ des résidents en fonction de l'année ${ }^{14}$. Pour la promotion 2016-2017, le nombre d'hommes ouvertement gay se chiffre à 10 sur environ 50 . En plus, quelques témoignages suggèrent que 
d'autres résidents hommes pourraient être homosexuels mais ne l'ont pas assumé ouvertement. Parmi le reste, la majorité des hommes adopte le discours égalitaire actuellement dominant au sein du milieu universitaire mexicain et des commentaires ou des comportements manifestement misogynes et homophobes n'ont pas lieu publiquement. S'il y a des résidents au comportement sexiste et homophobe, ils ne l'assument pas ouvertement.

Pendant cette première année de terrain 2016-2017 il y a eu une série de conférences animées par les résidentes autour des problématiques de genre (avortement, femmes à l'université, GPA, le rôle de femmes dans l'histoire du Mexique). Les conférences ont peu attiré l'attention des résidents, avec une minorité de résidents hommes dans le public. D'après ce que l'on a pu constater, une conscience et un niveau de réflexion relativement peu développé entourent cette question, sauf pour les résidentes conférencières sur l'avortement et la GPA, qui ont un point de vue construit à partir de leurs positionnements féministes.

11 Si l'échelle de recherche pour notre thèse va au-delà de la CIUP, à Paris et en région parisienne, cet article se concentre sur un groupe de jeunes hommes qui habitent dans la cité, et dont certaines caractéristiques clés sont résumées de façon anonymisée dans le tableau 1. Les faits qui y sont évoqués sont principalement issus d'un travail de terrain initial effectué en 2016-2017. Les critères de délimitation du terrain sont ceux proposés par l'anthropologue Michael Angrosino: premièrement, un endroit qui est susceptible de montrer le sujet de recherche choisi (les affects et le désir dans les trajets de mobilité des homosexuels Mexicains ayant vécu à Paris); deuxièmement : un site qui est comparable à d'autres déjà étudiés mais qui n’a pas été sur-analysé ; en troisième lieu, un endroit qui a peu de barrières d'entrée; enfin, un site dans lequel le chercheur ne représente pas une charge démesurée pour la communauté étudiée ${ }^{15}$.

Ces critères sont présents à Paris de façon générale, et, contrastent avec ceux d'autres pays européens, la centralisation française, le rôle de Paris dans l'imaginaire Mexicain, et la présence d'institutions comme la Maison du Mexique et l'Institut culturel du Mexique nous ont semblé pouvoir contribuer à la viabilité de la recherche. Non seulement on a pu trouver de nombreux sujets qui ont accepté de participer à l'enquête, mais on a même trouvé un groupe de mexicains homosexuels qui se fréquentent en raison du partage de la nationalité et d'une identification sexuelle. En ce moment, il n'y a aucune recherche publiée sur la migration homosexuelle mexicaine en Europe. Si les travaux sur les États-Unis sont nombreux, la seule autre destination à avoir été étudiée est la ville de Toronto par Rodrigo Laguarda ${ }^{16}$. Enfin, les enquêtés ont accepté de faire partie d'une observation ethnographique et de participer à des entretiens, octroyant leur confiance pour traiter d'un sujet qui est souvent classé comme appartenant à la vie intime, et seulement un a refusé de participer à un entretien formel.

\section{Enquêtés : critères d'inclusion}

En ce qui concerne les critères d'inclusion pour les enquêtés, il s'agit d'hommes mexicains habitant à Paris, arrivés entre 2008 et 2018, ouvertement homo- ou bisexuels. Toutes ces personnes font ou ont fait des études supérieures. Ils font partie des presque 100000 Mexicains qui vivent en Europe, dont 12800 en France selon des données de l'ONU pour l'année 2017, et ils font ou ont fait partie des 2514 Mexicains qui effectuent 
des études en France selon des données de l'UNICEF pour 2017 (avec une augmentation de $20 \%$ depuis 2013, la France étant la troisième destination pour les étudiants universitaires mexicains) ${ }^{17}$. Les premiers enquêtés ont été repérés dans des activités à la CIUP, et selon la méthode de la boule de neige, ils nous ont mis en contact avec d'autres homosexuels mexicains issus de leurs cercles sociaux. Même si l'on a pu rencontrer plusieurs femmes mexicaines bisexuelles ou lesbiennes, leur situation s'est avérée trop différente pour qu'on puisse l'inclure dans cette étude, au vu de l'ampleur qu'elle aurait dû prendre dans ce cas. Mais en effet, comme le dit Sandra Harding dans son livre Feminism and Methodology, une recherche féministe peut-être menée par des hommes et sans que l'on procède simplement à " rajouter des femmes »" . Il faut alors une «critique phallique ", c'est-à-dire un " examen critique des dimensions genrées des pensées et comportements masculins dans une perspective historique et interculturelle $»^{19}$, pour étudier des phénomènes comme les amitiés ou les rapports sexuels entre hommes, dans des contextes principalement masculins où une chercheuse rencontrerait des barrières d'accès importantes. ${ }^{20}$ Dans ce sens-là, il faut souligner une particularité de la migration mexicaine en Europe, celle de sa féminisation. En effet, selon les données de l'ONU transposées sur la carte ci-dessous, dans presque tous les pays européens, les femmes sont nettement plus nombreuses que les hommes, moyennant deux tiers du total en France et presque trois quarts en Italie. ${ }^{21}$

Figure 1 : Migrants mexicains en Europe

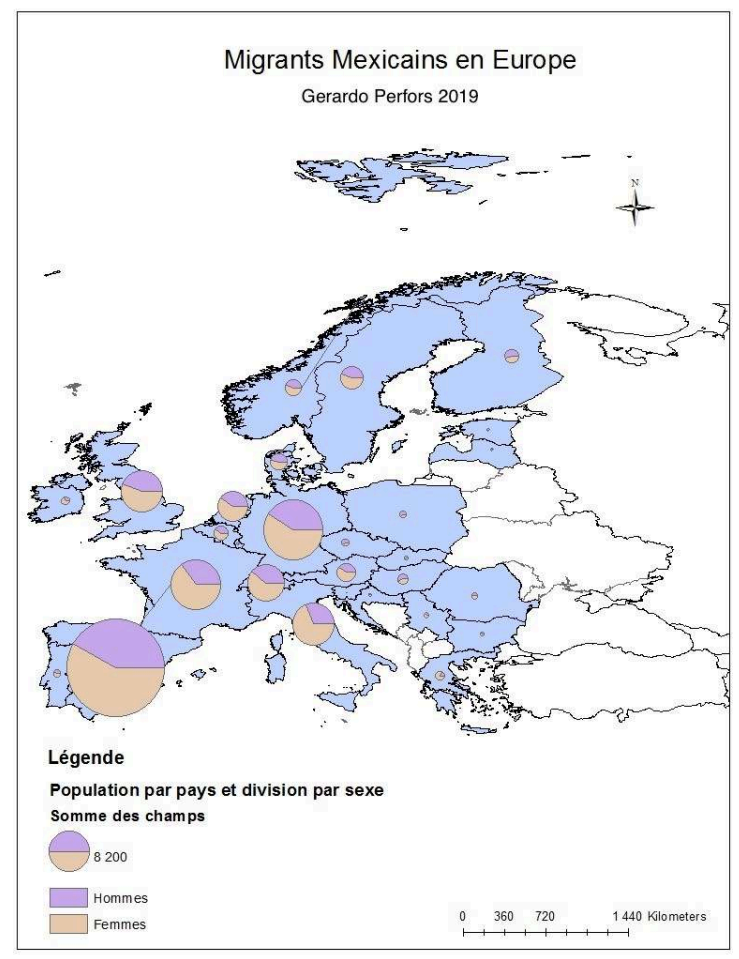

La taille des cercles reflète l'importance de la population mexicaine dans chaque pays par rapport au total européen de presque 100000 personnes. Les femmes sont nettement majoritaires dans les pays avec le plus grand nombre de migrants. Élaboration: Gerardo Perfors avec des données de I'ONU, 2019. 
Tableau 1. Aperçu du profil des enquêtés 


\begin{tabular}{|c|c|c|c|c|c|c|c|c|}
\hline Pseudo. & Origine & $\begin{array}{l}\text { Année de } \\
\text { naissance }\end{array}$ & Sexualité & Conjugalité & Occupation & Résidence & $\begin{array}{l}\text { Années à } \\
\text { l'étranger }\end{array}$ & $\begin{array}{l}\text { Exprime } \\
\text { le } \\
\text { souhait } \\
\text { de se } \\
\text { rapatrier }\end{array}$ \\
\hline Ernesto & Mexico & $80-85$ & Gay & $\begin{array}{l}\text { Couple } \\
\text { (Mexicain) }\end{array}$ & $\begin{array}{l}\text { Professeur } \\
\text { universitaire } \\
\text { / Doctorant } \\
\text { (Sciences } \\
\text { sociales) }\end{array}$ & $\begin{array}{l}\text { Mexico } \\
\text { (ex-CIUP) }\end{array}$ & $0-5$ & $\begin{array}{l}\text { Oui } \\
\text { (rentré) }\end{array}$ \\
\hline Javier & $\begin{array}{l}\text { Centre } \\
\text { du } \\
\text { Mexique }\end{array}$ & $85-90$ & Gay & Célibataire & $\begin{array}{l}\text { Masterant } \\
\text { (psychologie) }\end{array}$ & $\begin{array}{l}\text { Paris } \\
\text { (CIUP) }\end{array}$ & $0-5$ & Non \\
\hline Diego & Ouest & $85-90$ & Gay & Célibataire & $\begin{array}{l}\text { Org. } \\
\text { Internationale } \\
\text { Doctorant }\end{array}$ & $\begin{array}{l}\text { Paris (ex- } \\
\text { CIUP) }\end{array}$ & $0-5$ & Non \\
\hline Óscar & Côte sud & $85-90$ & Gay & Célibataire & $\begin{array}{l}\text { Employé (M2 } \\
\text { gestion) }\end{array}$ & $\begin{array}{l}\text { Paris } \\
\text { (CIUP) }\end{array}$ & $0-5$ & Indécis \\
\hline Sergio & Nord & $85-90$ & Gay & $\begin{array}{l}\text { Couple } \\
\text { (Français) }\end{array}$ & $\begin{array}{l}\text { Post-doc } \\
\text { (Sciences) }\end{array}$ & $\begin{array}{l}\text { Paris } \\
\text { (CIUP) }\end{array}$ & $5-10$ & Indécis \\
\hline Fernando & $\begin{array}{l}\text { Centre } \\
\text { du } \\
\text { Mexique }\end{array}$ & $85-90$ & Gay & $\begin{array}{l}\text { Couple } \\
\text { (Français) }\end{array}$ & $\begin{array}{l}\text { Employé } \\
\text { (informatique) }\end{array}$ & $\begin{array}{l}\text { Paris (ex- } \\
\text { CIUP) }\end{array}$ & $0-5$ & Non \\
\hline Lorenzo & Ouest & $85-90$ & Gay & Célibataire & $\begin{array}{l}\text { Doctorant } \\
\text { (SHS) }\end{array}$ & $\begin{array}{l}\text { Mexico } \\
\text { (ex-CIUP) }\end{array}$ & $0-5$ & $\begin{array}{l}\text { Oui } \\
\text { (rentré) }\end{array}$ \\
\hline Antonio & Mexico & $85-90$ & bisexuel & Célibataire & $\begin{array}{l}\text { Gérant } \\
\text { restaurant } \\
\text { (M2 gestion) }\end{array}$ & $\begin{array}{l}\text { Mexico } \\
\text { (ex-CIUP) }\end{array}$ & $0-5$ & $\begin{array}{l}\text { Oui } \\
\text { (rentré) }\end{array}$ \\
\hline José & Mexico & $85-90$ & Gay & Célibataire & $\begin{array}{l}\text { Employé CDD } \\
\text { (M2 LEA) }\end{array}$ & $\begin{array}{l}\text { Paris (ex- } \\
\text { CIUP) }\end{array}$ & $0-5$ & Indécis \\
\hline Poncho & Nord & $85-90$ & Gay & $\begin{array}{l}\text { Couple } \\
\text { (Français) }\end{array}$ & $\begin{array}{l}\text { Employé } \\
\text { (ingénieur) }\end{array}$ & Paris & $5-10$ & Non \\
\hline Carlos & $\begin{array}{l}\text { Mexico/ } \\
\text { Nord }\end{array}$ & $85-90$ & Gay & Célibataire & $\begin{array}{l}\text { Employé } \\
\text { (ingénieur) }\end{array}$ & Paris & $5-10$ & Oui \\
\hline Manuel & Sud-est & $90-95$ & Gay & Célibataire & $\begin{array}{l}\text { Étudiant } \\
\text { (arts) }\end{array}$ & Mexico & $0-5$ & $\begin{array}{l}\text { Indécis } \\
\text { (rentré) }\end{array}$ \\
\hline Héctor & Sud & $90-95$ & Gay & $\begin{array}{l}\text { Couple } \\
\text { (Français) }\end{array}$ & $\begin{array}{l}\text { Masterant } \\
\text { (SHS) }\end{array}$ & Banlieue & $0-5$ & Indécis \\
\hline
\end{tabular}




\section{Peur et espoir, désir et aversion : Réorientation des affects et pratiques incarnées}

14 La langue et la culture communes, leurs occupations, leurs problèmes communs en tant qu'étrangers qui ne connaissent pas les démarches pratiques face à la bureaucratie et la vie quotidienne en France, ainsi que leur niveau de revenus et leur localisation à Paris rassemblent un groupe d'hommes gay mexicains, qui se rencontrent souvent par l'utilisation d'applications de smartphones et ensuite constituent un groupe de socialisation distinct des autres groupes sociaux au sein des résidents mexicains de la CIUP, avec des activités à l'intérieur et à l'extérieur de la Cité. Les groupes sont préconstitués par les résidents des années précédentes qui intègrent les nouveaux arrivés.

15 Leurs corps subissent une réinterprétation en franchissant la frontière entre leur monde au Mexique et celui à Paris. Ceux qui ont des traits plutôt indigènes, habitués à une discrimination très répandue au Mexique ${ }^{22}$, découvrent souvent que ces mêmes traits sont désirables et populaires à Paris, même s'ils sont exotisés, et ils disent avoir beaucoup plus de "pegue" ${ }^{23}$ qu'au Mexique. Óscar, originaire d'une région du sud du Mexique, et ayant des origines indigènes, est celui qui se vante le plus de ses rencontres. "Fernando ${ }^{24}$ ? Celui qui vient de la région $* * * * * * 25$ ? Il m'a dit qu'il a énormément de succès à Paris ", me raconte un des enquêtés. En effet, tandis que Fernando se plaignait du " classisme » et du racisme au Mexique, il est actuellement en couple avec un Français. "Les gens à Puebla, où je faisais mes études, avaient des réactions très hostiles quand ils apprenaient d'où je viens. Je ne pourrai jamais retourner vivre dans cette ville.» D'autres, qui seraient vus comme «blancs » ou "plutôt blancs » au Mexique, et qui sont parfois issus de milieux privilégiés de classemoyenne supérieure, sont susceptibles d'être confondus avec des Arabes, comme c'est le cas pour Lorenzo ${ }^{26}$, fils de médecins issu d'une grande ville de province, qui a développé une aversion pour les Français et une préférence pour les hommes noirs et asiatiques depuis son arrivée à Paris.

16 Le séjour en France permet à certains de développer un sens de l'identité latinoaméricaine, comme c'est le cas pour Antonio ${ }^{27}$. Cette expérience en Europe développe également chez certains une division entre une identité latino-américaine/mexicaine vue comme «chaleureuse, gentille, amicale » face à l'identité européenne/parisienne vue comme «froide, distante » (Antonio) ou " pas généreuse », « malpolie » (Lorenzo).

On ajoute à cela l'éloignement des réseaux familiaux et amicaux du Mexique et les problèmes auxquels ils font face en tant qu'étrangers issus d'un pays du Sud. La gestion de la citoyenneté par l'État français, qui correspond à ce que le sociologue Lionel Cantú appelle "technologies de création de frontières ${ }^{28}$ détermine qui appartient pleinement à la France ou pas. Sergio ${ }^{29}$ en est le moins troublé, du fait de son statut professionnel de chercheur en sciences «dures" ainsi que par sa performance d'une masculinité «exotique » et "virile» valorisée en France. Óscar ${ }^{30}$, par contre, a dû passer quatre mois d'incertitude quant à son avenir professionnel et migratoire à la fin de son master en Gestion dans une école privée peu réputée. En revanche, remarque-til, une camarade Mexicaine a trouvé un emploi administratif dans une institution financière à l'issue du même master à Paris quelques semaines à la fin de son programme d'études. Au final, Óscar trouve un emploi pour un site de ventes par 
internet où il s'occupe du marché hispanique. Il y a une double conscience des défis auxquels ils ont dû faire face, d'un côté au Mexique, dans les domaines du travail, de l'éducation et du social, et puis en France en tant que non-citoyens racialisés devant étudier, travailler et socialiser dans une deuxième ou troisième langue. Ainsi, Sergio (le seul enquêté à avoir refusé de participer dans un entretien formel, souligne : "tu es européen, tu n'as pas besoin de penser aux choses sérieuses». En effet, étant un scientifique « dur ", il considère que les sciences sociales et humaines ne font pas partie des « choses sérieuses".

Si les difficultés et les défis rencontrés avant et après l'arrivée à Paris peuvent parfois mener à l'isolation ou à des conflits, ils peuvent simultanément contribuer à la création de liens affectifs et de soutien forts. En témoigne le rapport entre Antonio et Lorenzo par exemple. Lorenzo a accompagné Antonio à l'aéroport quand il rentrait au Mexique, et il a été accueilli chez lui quand il a été contraint à quitter la Cité suite à un désaccord avec la direction. Ernesto ${ }^{31}$ et Sergio s'entraident pour les procédures administratives et médicales: cela peut impliquer de passer une journée au commissariat pour accélérer les procédures d'émission du nouveau permis de séjour de Sergio; ou aller chez le médecin ensemble pour être sûrs d'avoir bien compris les diagnostics ou les traitements à suivre et de se soutenir si jamais il y a des mauvaises nouvelles.

Malgré des relations souvent difficiles, la distance physique avec la famille est mentionnée comme l'une des souffrances du séjour en France. Quand un camarade européen commentait qu'il lui paraît largement suffisant de voir sa mère deux fois par an, Sergio répond : «Ne dis pas ça si légèrement! Toi avec ta mère si près et les nôtres qui sont si loin !»; Ernesto ajoute : «oui, c'est dur, moi j'ai rêvé de ma mère l'autre nuit, je rêvais qu'elle me tenait dans ses bras!»; Javier ${ }^{32}$ avoue : "Je ne compte pas retourner au Mexique, mais c'est vrai que ma mère me manque. Même si elle me rendait fou très vite. »

20 Majoritairement originaires de villes moyennes de province, mais ayant fait leurs études de licence ou de master dans les plus grandes villes du pays, leur vie gay au Mexique était déjà développée, et ils indiquent souvent qu'ils avaient une meilleure qualité de vie au Mexique, en termes matériels et symboliques. Ainsi, un enseignant à l'université au Mexique devient un doctorant en France (Ernesto); un individu de classe moyenne-haute au Mexique, «juste» une personne quelconque à Paris (Lorenzo) ; un employé de bon niveau au Mexique, devient réceptionniste de nuit dans un hôtel à Paris (Antonio). Habitués des boîtes de nuit en vogue au Mexique avec une forte consommation d'alcool sur place, ils se retrouvent participants à des apéros dans des chambres d'étudiants ou se rendent à des fêtes gratuites, dans les bars du Marais à Paris (Antonio, Óscar). Ils expriment donc pour la plupart un désir de retourner au Mexique, qui est plus difficile à réaliser pour ceux qui ne sont pas issus des grandes villes. En effet, sur les treize enquêtés évoqués dans cet article, seulement quatre étaient rentrés au Mexique trois ans et demi après le début du terrain, et parmi ces quatre, trois étaient issus de Mexico et un d'une des trois autres grandes villes du pays, Guadalajara, Monterrey et Puebla. Indépendamment du retour ou non, le passage par Paris et leur inscription dans des établissements d'éducation supérieure parisiens accroît leur capital symbolique aux yeux de leurs compatriotes au Mexique, alors même que, selon eux, le niveau n'est pas toujours meilleur que celui des universités mexicaines (Óscar, Antonio, Lorenzo). "Je profite du malinchismo $»^{33}$, explique Fernando, informaticien. "L'équivalent du master que je fais ici m'aurait coûté 
beaucoup plus cher dans une université privée au Mexique, mais il ne m'aurait pas donné le prestige d'un diplôme français ».

21 Les enquêtés ne font pas partie de collectifs pour les droits LGBTQ, même si Fernando est membre d'un réseau de diplômés gays issus d'une grande université privée mexicaine. Ils voient des événements comme les marches des fiertés ou "prides" plutôt comme une occasion d'épanouissement social qu'un forum de revendications politiques.

Concernant l'implication politique au Mexique, le plus engagé est très impliqué dans les causes de la gauche souverainiste latino-américaniste, avec une conséquente production académique sur cet engagement (Ernesto). Deux autres se situent politiquement à gauche avec des expressions de soutien pour le parti Movimiento de regeneración nacional (MORENA) et son dirigeant Andrés Manuel López Obrador ${ }^{34}$, (Lorenzo, Antonio) ; deux autres sont beaucoup moins engagés (Sergio, Fernando), l'un prétend être d'une "droite conservatrice", exprimant son soutien à la femme de l'ancien président conservateur Felipe Calderón, mais de façon ironique, ce qui ne permet pas de juger de sa sincérité (Óscar).

On a pu identifier pendant le travail de terrain une série de signes de misogynie, qui se manifestent à plusieurs niveaux. Certains commentaires critiques de gays efféminés (Sergio), des insultes et des surnoms offensants au féminin "pendeja, chaira, lesbiana" (" co****se, gauchiste, lesbienne "; Lorenzo). La misogynie dans le langage des gays mexicains a été identifiée par List en $2002^{35}$, mais le classisme et le racisme identifiés par Laguarda chez ses enquêtés homosexuels mexicains à Toronto ne sont pas facilement repérés chez nos enquêtés à Paris ${ }^{36}$. Un autre commentaire frappant de la part de deux des enquêtés concernait la poitrine d'une des résidentes, et les avantages professionnels qu'elles lui conféreraient, et une série de hauts postes qu'ils pourraient atteindre s'ils en étaient dotés.

À plusieurs reprises, s'expriment une forte intolérance et méfiance envers la bisexualité d'Antonio, qui lui vaut le surnom de "lesbiana indecisa” («lesbienne indécise ») par Fernando et Lorenzo. Dans son entretien, Antonio déclare aimer les rapports avec les hommes et les femmes, affirme qu'il est gay, et se sent blessé par les réactions des autres :

También en un momento sentí mucha presión por parte de ellos de que es que tú tienes que definirte y no sé qué, y sentí un ambiente un poquito pesado, que dije, no sé si realmente esto es también lo que yo quiero, porque es un ambiente de, de puro sexo, de puro como cierto momento libertinaje, y que en su momento yo también dije, güey, tampoco esto es lo que estoy buscando. Entonces obviamente sí lo buscas, toda persona gusta del placer sexual, es muy normal, pero en cierto momento también me sentí muy muy presionado por parte de ellos de decir, tienes qué definir. Yo estoy muy bien definido y sé lo que me gusta y no. Yo no tengo que decir que soy gay porque realmente yo sé no soy gay. Si de verdad no me gustaran las mujeres diría que no me gustan pero sí me gustan. Y si dijera que no me gustan los hombres también mentiría. Entonces es algo que a veces no todos entienden, que simplemente lo tomo como si fuera un disfraz o una máscara, y a veces leí como en cierto momento como crisis de identidad, que ya tengo 28 años pero que aun así lo tienes, me informé, leí un libro y vi que algunas veces algunos bisexuales se ven atacados por ambas partes porque dicen que son gente no definida, y leí varios artículos y sí me sentí como identificado porque siento a veces la presión de mis amigos que los amo, los quiero, los adoro pero sí es la presión de esa parte. Entonces sí es complicado en ese sentido a veces. Un poquito complicado ${ }^{37}$. 
expérience de terrain nous a permis d'identifier une série récurrente de stéréotypes sexuels considérés comme attirants par les enquêtés, soit dans des conversations numériques groupées, soit dans des échanges, soit dans un photo-blog intitulé "la amante escondida", spécialement crée par un des membres pour télécharger des photos prises en cachette dans des endroits publics d'hommes qu'il considérait attirants. Dans ces conversations, Ernesto et Óscar mentionnaient leur appréciation des "chacales", un mot du vernaculaire mexicain pour parler d'hommes de classe ouvrière avec une virilité issue de leur travail physique. Lorenzo, comme on l'a dit précédemment, a développé une attirance pour les hommes d'origine africaine et asiatique, tandis que Fernando, qui alimente le photo-blog, aime des jeunes hommes barbus, musclés, soit en costume soit exerçant des métiers comme celui de policier, de maçon ou de pompier. Quant à Javier, le seul enquêté à avoir un phénotype européen, il exprime une préférence pour les Européens, à condition qu'ils ne soient pas efféminés.

Comme démontré par Lionel Cantú, cité précédemment, et confirmé par des chercheurs comme Héctor Carrillo ${ }^{38}$, la sexualité peut motiver la migration mais la migration modifie les pratiques et les imaginaires sexuels des migrants. Antonio explique que ses rapports sexuels "ont été différents ici en France, je pense que c'était avec le même nombre d'hommes que des femmes. Au Mexique c'était $80 \%$ des femmes et ici c'est fifty-fifty». Concernant l'origine de ces personnes: "Je ne sais pas si je vais tomber dans un autre stéréotype, diable! Mais ce qui est vrai c'est que en tant que latinos nous avons, nous sommes plus faciles, nous attirons plus facilement les Européens. Je n'ai été avec aucun asiatique, ni avec aucun noir, j'ai été avec des personnes européennes, honnêtement, et avec des Arabes des deux sexes ». Quand je lui demande s'il remarque des différences : "Et bien les yeux bleus, les güeritos [blonds/blancs], avant je n'aurais pas cru aimer les güeros aux yeux bleus avec mon préjugé sur comment les gringos sont un peu bêtes. Et puis tu te retrouves en Europe et c'est la première fois que je ressens une attirance pour ce type de personnes. Et voilà. " Comme pour Antonio, les rapports sociaux et sexuels interraciaux peuvent se révéler très compliqués pour la majorité des enquêtés. En effet, une grande majorité de Mexicains s'auto-identifie comme issue du métissage entre Espagnols et indigènes, cachant le fait qu'il existe, dans ce pays, une hiérarchie sociale en fonction de la tonalité de la peau et d'autres traits physiques comme la couleur des yeux et des cheveux, avec une valorisation des traits européens et un mépris pour l'héritage indigène ${ }^{39}$. Pour compliquer encore la situation, le genre et le sexe jouent un rôle important aussi dans ces interactions, puisque, dans un cadre (post)colonial, le mâle blanc se retrouve dans une position privilégiée structurellement, lui permettant d'exercer la violence sur les femmes racisées ${ }^{40}$. La totalité des implications de cette hiérarchisation dans les rapports homosexuels vont au-delà de la portée de cet article.

Pour Ernesto, enseignant dans une université au Mexique et doctorant à Paris, la migration a représenté une profonde mise en question de soi, vécue avec des moments d'insécurité profonde et avec une resignification des codes de socialisation et de drague. "No me siento en las mejores condiciones, no me siento tan seguro como en otros momentos" días que no pude expresarme en una tienda, y entonces me quedé sin comer algún día porque no me atreví a hablar de lo que quería comer y me regresé con toda la vergüenza del mundo, y regresé muy decepcionado." 42 Par ailleurs, il considère que la plupart de ses expériences sexuelles ont été "un sexo raro", et il lui semble que beaucoup d'hommes ont voulu avoir des rapports sans préservatif avec lui : "y entonces si la persona no me da confianza o, o 
siento que o veo que intenta como hacer algo sin condón, a mí se me pasa la emoción entonces ahí ya se acabó el asunto. No sé por qué. Y me ha pasado muchas veces." ${ }^{43}$ Même les différences perçues dans le croisement des regards produisent une expérience d'aliénation "en México es muy fácil ligar con la mirada. No sé, vas en la calle y ligas, y sientes una mirada, y si sientes una mirada hay una sonrisa. Y después de la sonrisa, ya tú decides si te paras en la banqueta, la otra persona se va a acercar o tú te vas a acercar. Y eso acá no he tenido mucho ese código. No sé si funciona igual, he sentido de repente algunas miradas en el metro en la calle, pero cuando yo volteo siento que cambian la mirada [... au Mexique ] no vas a tener esa negación al otro como de entrada." 44

À ce stade des recherches, le sujet des pratiques et représentations sociales, sexuelles et affectives des hommes enquêtés n'a pas été traité jusqu'à saturation. Par exemple, nous avons pu penser que les résidents homosexuels de la maison n'étaient pas attirés les uns par les autres. Or, les échanges avec Ernesto nous apprennent qu'il a eu des rapports avec l'un des résidents. Il s'agit d'un sujet tabou, peut-être, à cause de la proximité dans laquelle les enquêtés habitent les uns avec les autres et à cause du mélange entre liens d'amitié et liens d'attirance sexuelle ou romantique. Plus tard, on a appris que deux enquêtés avaient eu une liaison, dont l'attachement sentimental a perduré au retour de l'un des deux au Mexique. D'un côté, le fait d'habiter dans la même résidence rend les enquêtés très soucieux des regards et des jugements d'autrui, notamment en vue de l'homonormativitét5 qui n'est pas ouvertement remise en question par les enquêtés, à l'exception d'Ernesto.

\section{Des choses que l'on ne veut/peut pas savoir}

Godelier dit que la vie des gens est influencée par « des faits que l'on ne peut pas ou on ne veut pas savoir $\gg{ }^{46}$ Plus concrètement, des penseurs du XIX et du début du XX ${ }^{e}$ siècle comme Émile Durkheim, Karl Marx ou Karl Polanyi ont considéré que les grandes transformations sociales déclenchées à l'âge moderne donnent suite à des perturbations, qu'ils ont catégorisées comme "anomie», "aliénation», ou "dislocation ». Vers le milieu du $\mathrm{xx}^{\mathrm{e}}$ siècle, l'indépendance formelle de la majorité des pays africains et asiatiques et la mise en question des séquelles coloniales et impérialistes en Amérique latine ont été accompagnées d'une vague de réflexions et écrits autour de la condition postcoloniale. Selon ce courant analytique, l'indépendance formelle de la plupart des anciennes colonies n'a pas mis fin, pour autant, à ce que Walter Mignolo appelle "la dépendance épistémique», qui fait partie d'une "géopolitique de la connaissance » entre les métropoles occidentales et le reste du monde ${ }^{47}$. Parallèlement, la pensée féministe a permis une prise de conscience de l'oppression des femmes ainsi que le développement de la «critique phallique » dont on a parlé plus haut dans le texte.

En revanche, les publications et les débats publics concernant l'exclusion et les violences envers les homosexuels sont un peu plus récents, et se sont heurtés à un rejet social et institutionnel qui ne s'est affaibli qu'au cours des derniers trente ans, et cela dans la suite de l'épidémie du VIH/sida qui a pris la vie de milliers d'homosexuels, incluant des penseurs notables comme Michel Foucault et Guy Hocquenghem, pour ne citer que deux.

C'est donc dans ce processus partiel de prise de conscience et de mise en question des effets négatifs de la modernité sur les peuples, et plus spécialement sur les femmes, les 
descendants des peuples colonisés et les personnes qui ont des sexualités ou expressions de genre en dehors des normes, que ce groupe de jeunes homosexuels mexicains migre vers la France pour faire ses études. Ils tracent leurs trajectoires au sein d'un conflit discursif entre les récits idéalisés des vertus de la mondialisation, des droits humains et de la libération sexuelle, d'une part, et les violences structurelles qui excluent une majorité de la population mondiale de l'accès aux droits les plus élémentaires comme la santé, la sécurité, le logement, le travail et la libre expression. La migration, considérée comme «fait social total $»^{48}$ par le sociologue de la migration Abdelmalek Sayad ${ }^{49}$, dévoile certaines de ces contradictions que l'on ne pourrait ni l'on voudrait savoir autrement, et ceci pas seulement de façon théorique, mais de façon incarnée dans les corps et les expériences des migrants.

Ces faits peuvent nous dévoiler le fait qu'on est des citoyens abjects dans nos pays d'origine ou d'implantation. Ou bien, en fonction du milieu social de la personne, on n'est pas juste une victime de plus de l'exploitation coloniale mais plutôt une partie des agents qui participent au maintien de cet ordre.

"No me gustan los hombres mexicanos", raconte Javier, 32 ans, masterant en sciences sociales, de peau claire, aux yeux verts, et cheveux châtains, issu d'une famille de classe moyenne du centre du pays. "Por lo menos en mi ciudad, son todos iguales," pursuit-il. Quand on lui demande ce que cela veut dire, il explique, avec un peu de gêne: "Va a sonar racista, pero son gordos, morenos y chaparros" 50 . En effet, Javier préfère avoir des rapports avec des garçons plus grands que lui, pas ouvertement homosexuels, et avec un aspect physique typique du Nord de l'Europe. Il est l'un des seuls enquêtés à affirmer très clairement qu'il ne souhaite pas retourner au Mexique.

Dans un sens plus large, les participants s'interrogent par rapport à certaines des questions que l'on ne veut ou on ne peut pas poser, mais ils n'ont que peu d'outils ou de contextes dans lesquels ils pourraient répondre à ces interrogations. En effet, une chercheuse mexicaine a pu effectuer une recherche à la fin des années 2000, dans laquelle elle a interviewé la totalité des résidents de la Maison du Mexique, trouvant comme résultant une très haute fréquence de «crises émotionnelles, physiques, mentales, économiques et de valeurs » dont très peu osent parler ${ }^{51}$. Par ailleurs, lors de son terrain et pendant les entretiens, aucun des cent résidents n'a évoqué son homosexualité, hormis un jeune homme qui souffrait du rejet de son père à cause de l'association que ce dernier faisait de son choix d'être chef de cuisine et une supposée féminité ou homosexualité liées à ce métier.

Si même la majorité des étudiants et étudiantes mexicaines hétérosexuelles à Paris, qui pourraient être classés comme des privilégiés du fait de leurs origines dans les classes moyennes et leur accès à l'éducation supérieure, vivent ce type de crises profondes suite à leur migration, il devient peu surprenant que leurs camarades homosexuels aussi puissent rencontrer des conflits existentiels.

La distance géographique, économique et éducationnelle que les couches supérieures pourraient avoir avec les personnes les moins favorisées du Mexique ne leur permet pas toutefois d'échapper à la violence dans laquelle ces dernières vivent. Même de l'autre côté de l'océan, plusieurs d'entre eux gardent des souvenirs amers d'expériences de rejet à cause de leur sexualité ou de leurs origines sociales, et ils continuent à déployer des mécanismes d'autoprotection par peur de les revivre. José, diplôme d'un master en langues étrangères appliquées et employé en CDD, lors de son entretien, raconte qu'il ressent un malaise autour des hommes hétérosexuels mexicains, même 
ceux qui sont en France. Ernesto n'a que des relations ouvertes parce qu'il ne croit pas que la fidélité puisse exister. Quant à Sergio, on peut s'interroger sur ses remarques misogynes, qui lui évitent peut-être d'être vu comme un gay efféminé, plus susceptible d'être la cible des discriminations. Lorenzo évite d'avoir des relations sérieuses avec des personnes à Paris parce qu'il a décidé de rentrer au Mexique dès que possible ${ }^{52}$.Il est important de retenir que ces migrants s'étaient installés en Europe récemment, et ils étaient dans la plupart dans la "crise » identifiée par Laura Trejo dans son livre Crisis y transición: proceso de cambio en un grupo de estudiantes mexicanos en Europa, que l'on a cité précédemment.

\section{Le capital symbolique parisien}

Les difficultés liées à la migration et aux processus de discrimination qui vont avec peuvent être mieux comprises sous le prisme des idées de Pierre Bourdieu concernant le concept de capital symbolique, qui aide à comprendre de façon plus précise les raisons du choix de Paris et les conditions particulières inhérentes à ce choix. En effet, ce concept, défini comme « un capital de reconnaissance », « est une forme d'être perçu qui implique de la part de ceux qui perçoivent une reconnaissance de celui qui est perçu », « un statut social, une manière d'être social, d'être pour les autres ${ }^{53}$.

Prendre en considération le concept de capital symbolique nous permet de mieux comprendre les efforts des enquêtés pour éviter d'être infériorisés ou rejetés à cause de leurs origines ethniques ou sociales ou en raison de leur sexualité. Pour eux, faire des études supérieures à Paris serait une tentative « de changer l'être en changeant l'être perçu, dans la mesure où l'être perçu fait partie de la vérité complète de l'être quand il s'agit du monde social ${ }^{54}$. Ernesto, Javier et Óscar nous ont partagé des exemples de membres de leurs familles ou amis au Mexique qui sont devenus nettement plus admiratifs et affectionnés suite à leur installation à Paris, tandis qu'au Mexique ils étaient détachés et parfois même hostiles.

En revanche, dans le traitement et leurs expériences à Paris, il arrive que les enquêtés se sentent déclassés, du fait de leur aspect racisé, de leur usage du français en tant que langue étrangère, du manque de reconnaissance envers leurs trajectoires éducatives et professionnelles et du manque de prestige de la nationalité mexicaine vis-à-vis d'autres. La plupart d'entre eux avait un statut plus prestigieux au Mexique, ayant occupé des postes relativement importants avec des salaires qui leur permettaient de mener un style de vie qu'ils ne mènent pas à Paris en termes de consommation, de sorties et de logement, ou bien parce que leurs interactions sociales étaient plus satisfaisantes. Presque tous les enquêtés ont évoqué les difficultés qu'ils rencontrent dans leur vie à Paris qui les mènent à vouloir rentrer au Mexique ou bien à aller ailleurs: soit au niveau professionnel (impossibilité de trouver un emploi correspondant à leur diplôme : Óscar et Antonio; les collègues en CDI travaillent moins et ne sont pas accueillants: Sergio), économique (incapacité à trouver un logement dans Paris intra-muros: Javier) soit parce que leurs interactions affectives avec des Français ne sont pas satisfaisantes (ils seraient "froids », " distants », (Antonio) " pas généreux ", "malpolis » (Lorenzo) ou à cause de la mauvaise météo (" tout est gris, il n'y a jamais de soleil, il pleut tout le temps ": Lorenzo, Ernesto). Au niveau sexuel, cependant, on trouve que les trois enquêtés avec des traits typiquement indigènes (óscar, Fernando et Sergio) indiquent ce qui semblerait une amélioration vis-à-vis de 
leur situation au Mexique. En effet, l'enquêté qui est en France depuis le plus longtemps et qui s'est naturalisé français, Poncho vient d'une université privée très réputée au Mexique, où il a fait des études d'ingénieur qu'il a poursuivies en France, avec un nom de famille catalan, il a la peau légèrement matte, sans pour autant se faire typer racialement comme la majorité des enquêtés. Mesurant plus de $1,80 \mathrm{~m}$, il pratique plusieurs sports régulièrement. Il est en CDI depuis plusieurs années travaillant pour des grandes multinationales, et il est en couple avec un Français du même âge et qui est lui aussi diplômé de l'enseignement supérieur dans un secteur valorisé sur le marché du travail.

On perçoit alors que si certaines expériences négatives sont liées au contexte économique général des enquêtés en ce qui concerne l'emploi ou les loyers, ces problèmes ont empiré ou s'ajoutent à ceux qui émanent des questions du capital symbolique. Bourdieu affirme à ce sujet qu'«à l'origine de toute accumulation de capital symbolique il y a un fait arbitraire de nomination par lequel le groupe se constitue $»^{55}$. Une méthode d'exclusion consiste en la manipulation des frontières entre les groupes, et cette manipulation peut être « dure » (lois migratoires et attribution ou refus de visas, de permis de séjour) ou bien « douce »,(discrimination non-avouée dans l'attribution de logements, d'emplois, dans les relations sociales, etc. $\left.{ }^{56}\right)$. De plus, le contraste entre "le rêve parisien" existant dans l'imaginaire collectif mexicain, comme ville des arts, des intellectuels, et de l'amour, d'un côté, et la réalité de la migration et ses défis de l'autre rendent le sentiment de désillusion et de frustration plus fort pour des enquêtés comme Ernesto, Javier, Lorenzo et Óscar. «Quand tu vis au Mexique, on te vend Paris comme un rêve, magnifique, incroyable, ce qui est très difficile une fois que tu es sur place et que tu vois que ça ne correspond pas ", explique Ernesto. «C'est vrai », rajoute Javier. « Une fois, pendant mes vacances au Mexique j'ai vu une carte postale trop belle, et je me suis demandé où était cet endroit. En me rendant compte que c'était à Paris, je me suis dit : ça, ce n'est pas la ville où je vis !»

\section{Déplacement, reclassement ... dépassement ? Expériences imbriquées de la sexualité, de la race et de la classe auprès des membres du "Arcoíris en París"}

41 Revenant à la citation de Godelier sur l'objectif des sciences sociales, "d'analyser et comprendre les conditions d'apparition et de disparition des différentes façons d'organiser la vie en société ; des diverses manières de penser et d'agir, qui sont les racines de la diversité des formes connues d'identités individuelles et collectives, celles qui coexistent aujourd'hui et celles qui ont disparu », on constate que l'espace de la Cité Internationale Universitaire de Paris a été créé dans une société marquée par les hiérarchies de classe, de genre, de race et d'orientation sexuelle. Le plaisir et le désir incarnés des migrants mexicains qui y habitent se situent donc dans cette trame de domination. Le fait qu'on ait permis l'entrée de plus de femmes à la Cité et à la maison, que les gens soient catégorisés en hétéro- et homosexuels et que la tolérance envers ces derniers ait augmenté dans les dernières décennies tant en Europe qu'au Mexique a permis à plus d'hommes de s'affichent comme homosexuels et ce, à un âge plus jeune, $\mathrm{y}$ compris grâce à des applications de rencontre avec géolocalisation, qui permettent à leurs usagers de se repérer beaucoup plus vite et plus facilement qu'avant. Les jeunes mexicains de classe moyenne qui s'assument comme homosexuels ou bisexuels et qui 
intègrent le groupe "Arc-en-ciel à Paris ", compte tenu de leur diversité d'origines et de points de vue, réagissent différemment à ces conditions. Cependant, on retrouve chez la plupart d'entre eux le désir de retourner au Mexique une fois qu'ils auront mené à bien leurs études ou leur mission de recherche; ils pourront alors aspirer au capital symbolique du prestige colonial d'avoir vécu et étudié à Paris, mais aussi, pour certains, ils auront des points de vue plus critiques concernant les rapports internationaux qui pèsent sur leur mode de vie. Cependant, les probabilités du retour semblent beaucoup plus faibles pour les ressortissants des provinces, majoritaires dans notre échantillon. Cette particularité nous est devenue évidente dans une étape avancée du processus de rédaction, et mériterait une étude plus approfondie. Le désir, l'espoir, et la peur sont trois affects récurrents et jouent un rôle important dans la définition de leurs trajectoires. Et ces affects, semblerait-il, émanent des subjectivités individuelles des enquêtés ainsi que du contexte et des conditions de leur migration. La traversée des frontières ainsi que la resignification de leurs corps et de leurs comportements dans leur nouveau contexte parisien reterritorialise leurs repères culturels et sexuels, leurs affects, et leur positionnement face au monde.

\section{NOTES}

1. Maurice Godelier, Au fondement des sociétés humaines: ce que nous apprend l'anthropologie, Paris, Flammarion, 2010, p. 244.

2. Richard G. Parker, Beneath the equator: cultures of desire, male homosexuality, and emerging gay communities in Brazil, Londres \& New York, Routledge, 1999.

3. John D'Emilio, “Capitalism and Gay Identity”, in Ann Barr Snitow, Christine Stansell (dir.), Powers of Desire: The Politics of Sexuality, Monthly Review Press, 1983.

4. Selon des données de l'Organisation Internationale du Tourisme de l'ONU, le nombre annuel de touristes est passé de 25,2 millions en 1950 à 1,4 milliards en 2018 : United Nations World Tourism Organization, “UNWTO World Tourism Barometer 2019", UNWTO World Tourism Barometer, 2019.

5. "Vêtues ", un des différents mots péjoratifs utilisés pour se référer aux personnes avec des sexualités ou genres non-normatifs: Michael Schuessler, "Vestidas, Locas, Mayates' and 'Machos': History and Homosexuality in Mexican Cinema”, Chasqui, $\mathrm{n}^{\circ} 34$, 2005, p. 132.

6. Carlos Monsiváis, Que se abra esa puerta: crónicas y ensayos sobre la diversidad sexual, Paidós Mexicana, 2010; Rodrigo Laguarda, De sur a norte: chilangos gays en Toronto, Instituto Mora: CONACYT, 2014.

7. Cymene Howe, "Sexual Borderlands: Lesbian and Gay Migration, Human Rights, and the Metropolitan Community Church", Sexuality Research and Social Policy, vol. 4, $\mathrm{n}^{\circ} 2$, juin 2007, p. 88-106; Kath Weston, Families We Choose: Lesbians, Gays, Kinship, Columbia University Press, 1997. 
8. Sandra G. Harding (dir.), The feminist standpoint theory reader: intellectual and political controversies, Londres, Routledge, 2004.

9. Charlotte Aull Davies, Reflexive ethnography: a guide to researching selves and others, Londres, Routledge, 2008; Louis Dupont, «Terrain, réflexivité et auto-ethnographie en géographie ", Géographie et cultures, $\mathrm{n}^{\circ}$ 89-90, mars 2014, p. 93-109; Rosana Gúber, El salvaje metropolitano: reconstrucción del conocimiento social en el trabajo de campo, Editorial Paidós, 2004; Marianne Morange, Camille Schmoll, Étienne Toureille, Les outils qualitatifs en géographie : méthodes et applications, Malakoff, Armand Colin, 2016.

10. Charlotte Aull Davies, Reflexive ethnography: a guide to researching selves and others, Londres, Routledge, 2008, p. 18.

11. Francisco Colmenares, "Petróleo y crecimiento económico en México 1938-2006", Economía UNAM, $\mathrm{n}^{\circ} 15$, décembre, 2008.

12. «Témoignage perdurable de l'amitié entre le Mexique et la France, ce bâtiment construit pour la jeunesse studieuse.»

13. Sergio Zermeño, Las trampas de la belleza, Porrúa, 2014, p. 10.

14. Nous avons effectué une enquête auprès d'anciens résidents homosexuels et hétérosexuels de la Maison qui y ont résidé entre les années 1980 et la première décennie des années 2000, qui confirme que le nombre de résidents ouvertement homosexuels était entre un ou deux maximum, ce qui est nettement inférieur à celui observé sur le terrain entre 2016 et 2019.

15. Michael V. Angrosino, Doing Ethnographic and Observational Research, Londres, Sage Publications, 2008, p. 29-30.

16. Rodrigo Laguarda, De sur a norte: chilangos gays en Toronto, Instituto Mora, CONACYT, 2014.

17. UNESCO. Migration Profile: Mexico, https://esa.un.org/miggmgprofiles/indicators/files/ mexico.pdf, consulté le $1^{\text {er }}$ décembre 2017; ONU. Trends in International Migrant Stock: the 2017 Revision, http://www.un.org/en/development/desa/population/migration/data/estimates2/ estimates17.shtml, consulté le $1^{\text {er }}$ décembre 2017.

18. Sandra Harding, Feminism and Methodology, Indiana University Press, 1987, p. 3.

19. Ibid. p. 12.

20. Ibid., p. 11-12.

21. Pour plus de détails sur la migration féminine du Mexique vers l'Europe, consulter : Paulina Sabugal, "Amor e identidad. El caso de la migración mexicana en Italia", Antropología Americana 3, $\mathrm{n}^{\circ}$ 5, 2018, p. 73-87.

22. Luis Soberanes Fernández, "Igualdad, discriminación y tolerancia en México", Cuestiones constitucionales, $\mathrm{n}^{\circ} 22$, juin, 2010, p. 261-274.

23. « Du succès ».

24. Fernando, né entre 1985-1990, issu de la région de la Vallée du Mexique, gay, en couple avec un homme français.

25. État rural et avec une importante population indigène près de la ville de Mexico, dont le nom est omis pour maintenir l'anonymat de l'enquêté.

26. Lorenzo, né entre 1985-1990, issu d'une grande ville dans l'ouest du Mexique, gay, célibataire.

27. Antonio, né entre 1985-1990, issu de Mexico, bisexuel, célibataire.

28. Lionel Cantú et al., The sexuality of migration: border crossings and Mexican immigrant men, New York University Press, 2009.

29. Sergio, né entre 1985-1990, issu du Nord du Mexique, gay, en couple avec un homme français.

30. Óscar, né entre 1985-1990, issu de la côte sud du Mexique, gay, célibataire. 
31. Ernesto, né entre 1980-1985, issu de Mexico, gay, en couple avec un homme Mexicain au Mexique.

32. Javier, né entre 1985-1990, issu d'une ville dans la région de la Vallée du Mexique, gay, célibataire.

33. Terme mexicain qui désigne la croyance que tout ce qui vient de l'Occident est intrinsèquement meilleur que ce qui vient du Mexique.

34. Ce dirigeant, élu président en juillet 2018, et son parti, devenus majoritaires au Congrès dans les mêmes élections, étaient dans l'opposition lors de la rédaction de cet article.

35. Mauricio List Reyes, "La noche de El Ansia", ALTERIDADES, vol. 12, n 23, 2002, p. 63-81.

36. Rodrigo Laguarda, De sur a norte: chilangos gays en Toronto, Instituto Mora, CONACYT, 2014.

37. «Aussi, parfois, j'ai senti une grande pression de leur part pour me définir, et j'ai ressenti une ambiance un peu lourde, et je me suis dit, « je ne suis pas sûr que ceci soit ce que je veux, car c'est une ambiance où il n'y a que du sexe, que des moments de libertinage, et que, à un moment donné, moi-même je me rendais compte que ce n'est pas ce que je recherche. Alors évidemment tout le monde aime le plaisir sexuel, c'est très normal, mais dans un certain sens je me suis senti dans la contrainte de me définir. Je suis très bien défini et je sais ce qui me plaît et ce qui ne me plaît pas. Et je ne vais pas dire que je suis gay car vraiment je sais que je ne suis pas gay. Si je n'aimais pas les femmes en vrai, je le dirais, or je les aime bien. Et si je disais que je n'aime pas les garçons alors je mentirais aussi. Donc, c'est quelque chose que pas tout le monde comprend, comme si j'avais un déguisement ou un masque, et alors j'ai cru avoir une crise d'identité, que même à 28 ans cela peut arriver. Je me suis renseigné, j'ai lu un livre et j'ai vu que parfois certains bisexuels se font attaquer des deux côtés car on dit que ce seraient des gens indéfinis, et j'ai lu plusieurs articles. Je m'y suis identifié car je ressens parfois la pression de mes amis, même si je les adore, je les aime, mais ils me mettent une pression en ce sens-là. Alors oui c'est compliqué à cet égard parfois. Un peu compliqué. »

38. Héctor Carrillo, Pathways of desire. The sexual migration of Mexican gay men, Chicago, The University of Chicago Press, 2017.

39. Rosario Aguilar, "Los tonos de los desafíos democráticos El color de la piel y la raza en México", Política y gobierno 20, nº 3, septembre 2017, p. 25-57.

40. Pascal Blanchard et al. (dir.), Sexe, race \& colonies, Paris, Éditions La Découverte, 2018 ; Frantz Fanon, Peau noire, masques blancs, Chicoutimi, Université de Québec à Chicoutimi, 2015.

41. «Je ne me sens pas dans les meilleures conditions, je ne me sens pas sûr de moi-même comme j'ai pu me sentir dans d'autres moments. »

42. "Je croyais que j'allais bien, et alors je suis venu et je me souviens qu'il y a même eu un jour où je n'ai pas pu m'exprimer dans un magasin, et alors je n'ai pas pu manger ce jour-là car je n'ai pas osé dire ce que je voulais manger et je suis rentré chez moi avec toute la honte du monde, et je suis rentré avec une grande déception. »

43. «Et alors, si la personne ne me semble pas fiable, ou bien si je ressens qu'elle veut faire quelque chose sans préservatif, je perds l'envie et c'est mort. Je ne sais pas pourquoi. Et cela m'est arrivé plusieurs fois. »

44. "Au Mexique c'est très facile de draguer avec le regard. Je ne sais pas, tu vas dans la rue et tu dragues, et tu ressens un regard, et alors un sourire. Et après le sourire, c'est à toi de voir si tu t'arrêtes sur le trottoir, si l'autre t'approche ou si toi tu l'approches. Et je n'ai pas trop eu ce code ici. Je ne sais pas si ça marche pareil. J'ai ressenti quelques regards dans le métro, dans la rue, mais après ils regardent ailleurs. [... au Mexique] il n'y a pas cette négation d'autrui du départ. » 
45. "Dominant assumptions of the normalcy and naturalness of monogamy, analogous to such assumptions around heterosexuality inherent in the term heteronormativity", in Meg Barker, "Whatever happened to non-monogamies? Critical reflections on recent research and theory", Sexualities, $\mathrm{n}^{\circ} 6$, décembre, 2010, p. 750.

46. , Maurice Godelier, Au fondement des sociétés humaines: ce que nous apprend l'anthropologie, Paris, Flammarion, 2010, p. 250.

47. Walter Mignolo, "Cambiando las éticas y las políticas del conocimiento: lógica de la colonialidad y postcolonialidad imperial", Tabula rasa, $\mathrm{n}^{\circ} 3,2005$, p. 47-72.

48. Selon l'anthropologue Marcel Mauss dans son classique Essai sur le don, un fait social total a la particularité de mettre « en branle dans certains cas la totalité de la société et de ses institutions", Marcel Mauss, Essai sur le don, Chicoutimi, Université de Québec à Chicoutimi, 2002, p. 102.

49. Abdelmalek Sayad, L'immigration ou les paradoxes de l'altérité. L'illusion du provisoire, Paris, Raisons d'Agir, 2006.

50. «Je n'aime pas les hommes mexicains. Dans ma ville, au moins, ils sont tous pareils. Ça va avoir l'air raciste, mais ils sont gros, petits et bruns. »

51. Laura Trejo, Crisis y transición: proceso de cambio en un grupo de estudiantes mexicanos en Europa Monterrey, Mexico, Universidad Autónoma de Nuevo León, 2011.

52. En ce sens, une hypothèse qui pourrait être intéressante à explorer dans des recherches à venir concerne la pertinence du phénomène que Vilfrido Pareto identifie comme le «dépérissement des élites » dans le cas du comportement de certains hommes gays. Pierre Bourdieu décrit ce dépérissement quand les membres d'élites «sont conduits au déclin par une sorte de conscience malheureuse de leur propre domination qui leur interdit de s'assumer comme dominants et les conduit à accepter sur eux-mêmes le point de vue des dominés ». Pierre Bourdieu et al., Sociologie générale, Paris, Raisons d'agir, Seuil, 2015, p. 187.

53. Ibid. p. 130-132.

54. Ibid.

55. Ibid.p. 136.

56. Ibid. p. 136, 138.

\section{RÉSUMÉS}

La resignification des repères corporels, sociaux, ethniques et sexuels d'un groupe de Mexicains homosexuels à Paris au long de leurs trajectoires de mobilité, ainsi que le rôle des affects et du désir dans l'incorporation de ces déplacements fait l'objet de ce travail. Le déplacement vers une société avec des notions de la sexualité et de la race différentes mène à un (auto) reclassement, avec des résultats souvent inattendus. Un problème commun évoqué par les enquêtés est la tension entre différents types d'exclusion dans leur société d'origine et dans leur ville d'accueil. Cet article reflète le progrès d'une thèse de doctorat en cours, avec une approche (auto)ethnographique, réflexive, féministe. 
La resignificación de las coordenadas corporales, sociales, étnicas y sexuales de un grupo de mexicanos homosexuales en París, a lo largo de sus trayectorias de movilidad, así como el papel de los afectos y el deseo en la incorporación de dichos desplazamientos, forman el objeto de este trabajo. El desplazo a una sociedad con nociones de la sexualidad y la raza diferentes lleva a una (auto) reclasificación, con resultados a veces inesperados, Un problema común evocado por los informantes es la tensión entre diferentes tipos de exclusión, tanto en su sociedad de origen como en su ciudad destino. Este artículo refleja el progreso de una tesis doctoral en curso, y se apoya en un enfoque (auto)etnográfico, reflexivo y feminista.

\section{INDEX}

Mots-clés : migration, affects, homosexualité, Mexique, Paris

Palabras claves : migración, afectos, homosexualidad, México, París

\section{AUTEUR}

\section{GERARDO PERFORS-BARRADAS}

Laboratoire ENeC, Sorbonne Université 